\title{
Ophthalmoscopic appearances after injury to the circle of Zinn
}

\author{
ANWAR ELMASSRI \\ Ophthalmic Department, Ein-Shams Faculty of Medicine, Cairo University, Egypt
}

Concussion injury to the globe, if not severe enough to rupture the outer coat, causes a temporary expansion of the posterior sclera. This may pass unnoticed without producing any lesion, or may be severe enough to rupture the adjacent vascular structures. The circle of Zinn, by its particular situation around the optic nerve and within the sclera, is liable to such injury and its rupture is a possible cause of clinical appearances seen in some of the following eight cases. In the other cases there is probably a similar lesion of a short posterior ciliary artery or cilio-retinal vessel.

\section{Case reports}

(I) A young man aged 22 years received a direct blow on the left eye from a book, and at once noticed blurring of the central vision. The case was diagnosed as one of central retinal detachment with a possible macular tear. When I saw him 3 days after the accident there appeared to be a choroidal haemorrhage around the disc involving the macula.

Ophthalmoscopically a dark area intermingled with a dull-greyish elevation encircled the disc which was of a normal colour, and extended on the temporal side to reach the central area. The retinal vessels were running normally over the elevation and there was no sign of involvement of the overlying retina. The central vision was $\mathrm{i} / 6 \mathrm{o}$.

Conservative treatment in the form of rest and vitamin $\mathrm{C}$ was advised, but after one month there was no improvement of vision, which remained I/6o with a marked scotomatous temporal field defect. The ophthalmoscope now showed that the dark and greyish elevation had absorbed, leaving an irregular peripapillary fibrosis with an intact overlying retina and retinal vessels (Fig. I, col. pl.). The optic disc showed typical atrophic changes.

FIG. I Irregular peripapillary thbrosis. Optic disc atrophic (Case I).

FIG. 2 Peripapillary pigmentation with choroidal atrophy after removal of intraorbital tumour (Case 2).

FIG. 3 Well defined choroidal scar bespattered with pigment below atrophic disc (Case 3).

FIG. 4 Triangular pigmented patch with its base at the temporal disc margin, with an attempt to encircle the disc on the nasal side (Case 4).

FIG. 6 Area of irregular choroidal pigmentation on temporal side of left disc (Case 6).

FIG. 9 Linear area of choroido-retinal atrophy extending from the disc margin to the macula in the classical position of a cilio-retinal vessel (Case 8 ).

Received for publication July 2, 1970

Address for reprints: Ophthalmic Department, Ein-Shams Faculty of Medicine, Cairo University, Egypt 
Ophthalmoscopic appearances after injury to the circle of Zinn
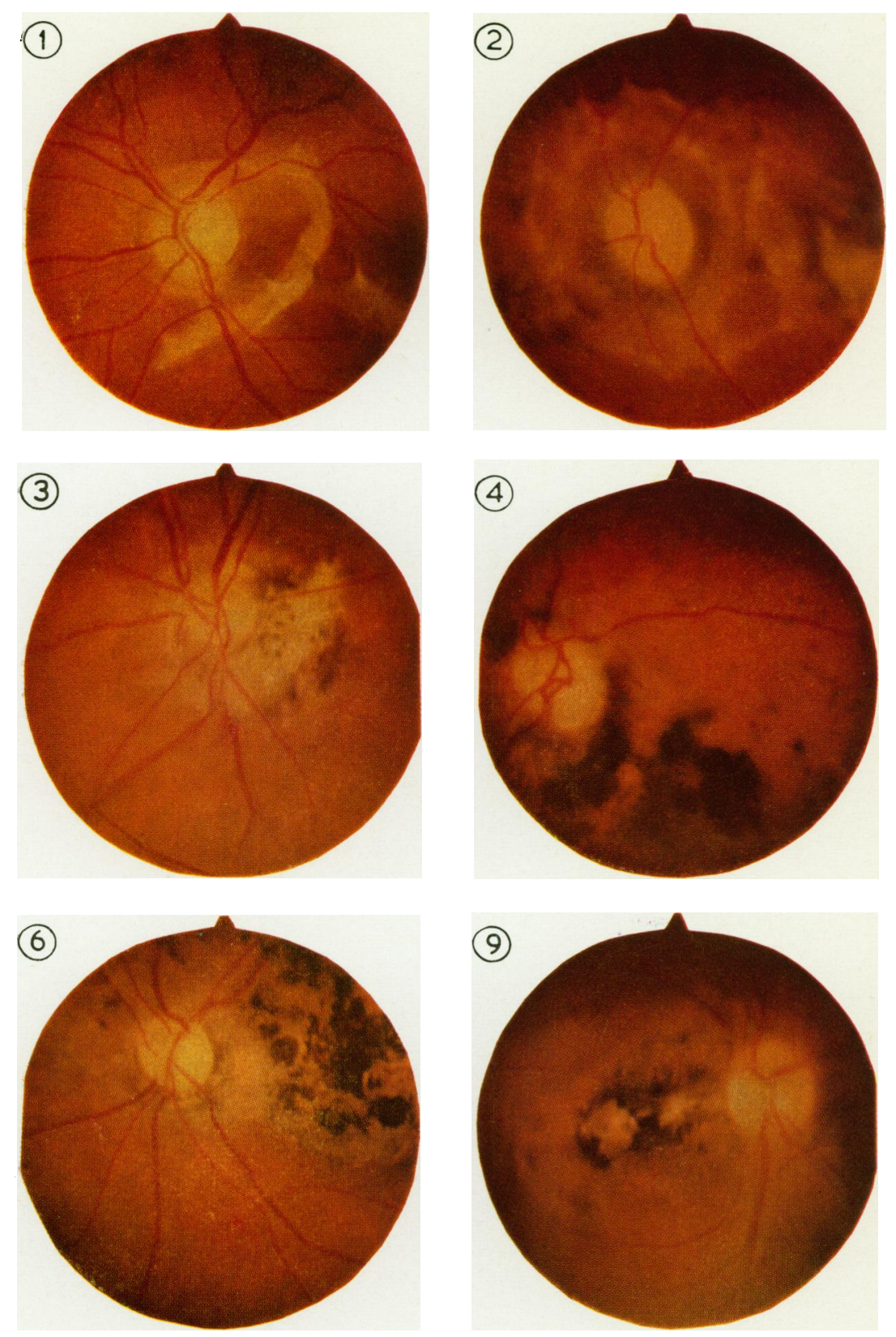

To face page 12 . 
(2) A young girl aged 18 years had had a successful removal of a seemingly benign orbital mass. One month later, when the eye was uncovered, she noticed marked diminution of vision, which was $1 / 60$ with scotomatous defects in the nasal and temporal fields.

Ophthalmoscopic examination revealed a peripapillary greenish-grey pigmentation (Fig. 2, col. pl.). The retinal vessels passed normally over the lesion but the macular area was encroached upon. The disc showed a slight degree of atrophy. The diagnosis was peripapillary choroidal atrophy and fibrosis after a haemorrhage resulting from compression of the globe during the removal of the orbital mass.

(3) A man aged 35 years, during a routine refraction, was found to have had defective vision in the left eye for several years. He gave a history of a blunt injury to this eye ro years before, after which he had had a rapid diminution of vision. No treatment was effective at the time.

Ophthalmoscopically a well-defined scar area bespattered with pigment was detected below an atrophic disc. There was a narrow extension of the scar around the disc on its upper margin (Fig. 3, col. pl.). The retinal vessels passed uninterruptedly over the affected area.

(4) A woman aged 23 years complained of marked diminution of central vision which was found to be counting fingers at $1 \mathrm{~m}$. The nasal field was markedly affected and there was a tendency to diverge on occlusion of the eye. The left eye was normal. She stated that she had suffered a direct blow on the right eye by a blunt object 5 years before. Immediately after the accident she had noticed blurring of vision. No treatment was effective, and the vision gradually deteriorated.

Ophthalmoscopic examination revealed a triangular patch with masses of pigment, the base being at the lower temporal quadrant of a pale disc and the apex towards the periphery. There was a smaller pigmented area at the opposite edge of the disc and the macular region was besprinkled with pigment (Fig. 4, col. pl.). The retinal vessels passed uninterruptedly over the lesion.

(5) A young man aged 18 years received a direct hit on the left eye while playing football. As the blurring of vision which followed the injury was slight he did not trouble to consult a specialist, but he noticed that the visual acuity of the two eyes was gradually becoming unequal. The acuity of the right eye was $6 / 6$ and that of the left $6 / 18$ one year after the accident.

Ophthalmoscopic examination revealed a well-defined irregularly-pigmented atrophic patch close to the upper temporal margin of the disc. The retinal vessels passed uninterruptedly over the patch. The colour of the disc was normal (Fig. 5).

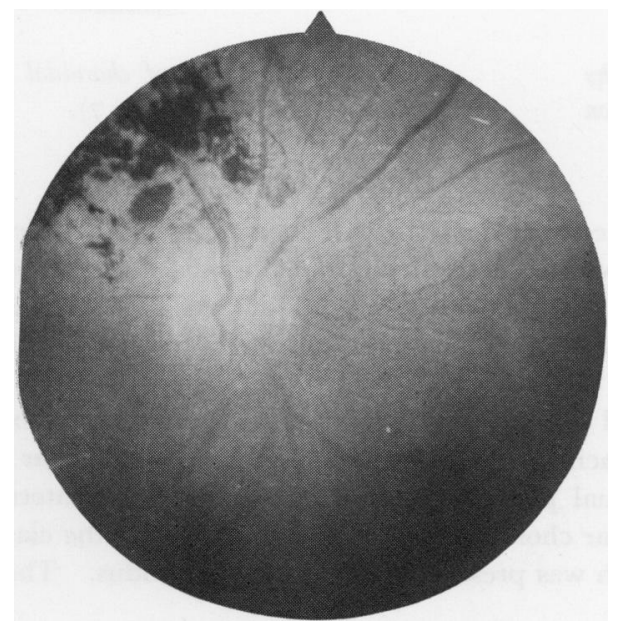

FIG. 5 Well-defined irregularly-pigmented atrophic patch close to upper temporal margin of disc (Case 5). 
(6) A boy aged 12 years was found to have weak vision in the left eye (6/6o with correction) at a routine refraction. He was a high hypermetrope, about $+6 \mathrm{D}$ sph. in both eyes. His parents said he had suffered a direct hit on the left eye by a tennis ball one year before. He had had only slight blurring of vision and treatment had been concentrated on the chemotic lids and conjunctiva.

Ophthalmoscopic examination revealed a well-defined round area of irregular pigmentation on the temporal side of the disc, extending to the macular region. Similar pigmented clusters were observed on the upper edge of the disc and there was a halo of choroidal atrophy surrounding the atrophic optic nerve head. The retinal vessels passed normally over the affected region (Fig. 6, col. pl.).

(7) A young man aged 25 years was refused a job because of weak vision in the right eye, I/6o with eccentric fixation and field defects especially temporally and downwards. He said he had suffered a blow on this eye by a blunt object 5 years before.

Ophthalmoscopic examination showed a well-defined ring of peripapillary choroidal atrophy bespattered with a dark-greenish pigmentation. The ring was irregularly placed around the disc, extending more on the temporal side, less on the inferior margin, and much less on the upper nasal border. There was a tongue-shaped extension towards the macula which showed a marked stippling (Figs 7 and 8). The retinal vessels passed normally over the affected area. The remaining fundus showed no such patches.

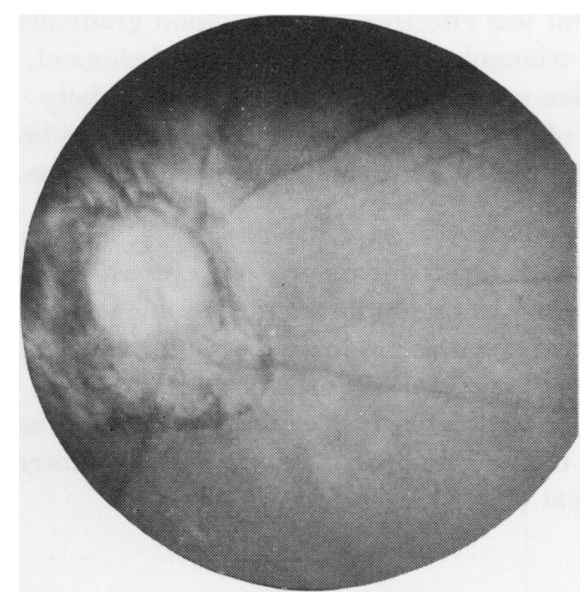

FIG. 7 Ring of peripapillary choroidal atrophy besprinkled with dark-greenish pigmentation (Case 7).

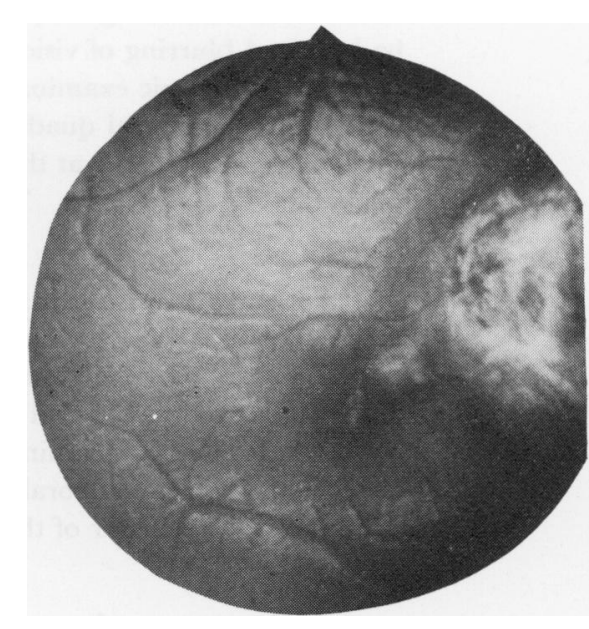

FIG. 8 Extension of choroidal ring of Fig. 7 towards the macula (Case 7).

(8) A woman aged 30 years complained that the left eye tended to diverge. The visual acuity in this eye was counting fingers at $\mathrm{I} \mathrm{m}$. with eccentric fixation. She said that $\mathrm{Io}$ years before she had received a blow from her child's fist, and that this had been followed by blurring and gradual diminution of vision, which did not respond to treatment. Central choroiditis was the provisional diagnosis but no cause was found.

Ophthalmoscopic examination revealed a linear area of choroido-retinal atrophy extending from the temporal margin of the disc to the macula. The area was fringed with irregular pigmentation which divided the patch into nearly equal parts. A retinal artery passed uninterruptedly over the upper border of the lesion. The linear chorio-retinal scar was situated in the classical position of a cilio-retinal artery. No similar patch was present in the rest of the fundus. The disc showed some degree of atrophy (Fig. 9, col. pl.). 


\section{Discussion}

The short posterior ciliary arteries as they pierce the sclera give branches which anastomose to form an arterial circle within the sclera around the optic nerve head. This circle of Zinn-Haller may also receive contributions from the most distal portion of the pial arterial plexus. Rarely, the circle of Zinn may be replaced by another circle placed slightly posteriorly within the dura. The circle of Zinn gives out branches to the pial sheath of the optic nerve, to the region of the lamina cribrosa thus contributing to the vascularization of the optic nerve head, and branches which pass into the peripapillary part of the choroid where they become associated with the small branches of the short posterior ciliary arteries which turn back into this region from the main part of the choroid. The cilio-retinal artery, which appears ophthalmoscopically at the margin of the disc and usually on its temporal side, is an inconstant branch of the circle of Zinn. It is concerned with the supply of a portion of the retina between the disc and the macula. Although the anatomical evidence in the literature of the existence of the cilio-retinal artery is scanty, Wybar ( 1954) demonstrated it in a neoprene cast. Together with the circle of Zinn, the posterior choroid is supplied by certain ciliary arteries which, instead of passing forwards, terminate near the site of perforation by the dichotomous formation of arterioles which finally break up to form the choriocapillaries. As for the segmental localization within the choroid, it has been suggested that distinct specialized zones are present. These constitute the equator, the periphery, the submacular, and the peripapillary zone (Wybar, 1954). This probably applies to the arteries which run in sectors without anastomosing with their neighbours, but the continuity of the choriocapillaries over the entire choroid is complete. A continuous network in the peripapillary zone, composed of arterioles which do not participate in the vascular supply of the rest of the choroid, was described by Marin-Amat (Discussion of Bartolozzi, I 952).

The first mention of a lesion of a short posterior ciliary artery was made by Coats (1907), who referred to a case reported by Knapp ( I868) of a woman suffering from mitral regurgitation and cardiac hypertrophy who experienced failure of vision in both eyes after two attacks of giddiness. In the first attack there was a diffuse greyish opacity in the right retina around the macula and below the nerve; in the second attack a retinal opacity was found above the left macula. Both retinal lesions cleared up in 3 to 5 days. Knapp's explanation of the phenomena was that on both occasions the endocarditis had given rise to emboli which had lodged in some vessel of the brain and also in the posterior ciliary arteries. It is more likely that the condition was a spasm of branches of the central retinal artery; the part played by the short posterior ciliary arteries is rather controversial. Coats (1907) also described the histopathological appearance of an eye removed as a result of iridocyclitis with secondary glaucoma; because of the presence of extensive necrosis and inflammatory infiltration of the sclera, choroid, and retina, he concluded that the patient's symptoms were best explained as an obstruction of a posterior ciliary artery with a consequent infarction of the inner layers of the sclera, choroid, and retina. There was no history of trauma in either case.

Bartolozzi (1952) presented a case of post-traumatic peripapillary choroidal atrophy which he attributed to rupture of the finest arterial branches of the posterior ciliary arteries. In the ensuing discussion Marin-Amat (1952) agreed with the diagnosis and suggested that the peripapillary choroidal atrophy was pathognomonic of deficient circulation in that region. According to him the arborization of the short posterior ciliary arteries nearest to the papilla resolves into a continuous arterial network which supplies 
blood to the peripapillary zone of approximately the diameter of the papilla. He concluded that the forward stretching of the eyeball as a result of posterior trauma explained the rupture of the fine branches of the short posterior ciliary arteries. A torsion of the eye resulting from such trauma was suggested by Bartolozzi (1952).

By the "continuous arterial network", Marin-Amat probably meant the circle of Zinn.

In discussing the sequelae of contusion of the posterior pole on the papilla, Bonamour, Brégeat, Bonnet, and Juge ( 1968 ) stated that ophthalmoscopically their intensity paralleled that of the primary papillary and peripapillary manifestations. The papilla usually retains a normal aspect, but when the trauma is severe a cicatricial peripapillary degeneration appears in the subsequent months. In its early form, this degeneration is seen as a $\overrightarrow{0}$ ring of pigment with bluish reflexes around the disc associated with a semi-annular para- $\overrightarrow{\vec{H}}$ central scotoma. At a later stage pigment migration is associated with a band of a peripapillary atrophy. The pigment is gathered at the centre of this band or disposed on its edges. No explanation is given to this clinical picture or how it is developed.

Duke-Flder (1954) mentioned a clinical picture wherein a spherical, yellowish area with :well-defined margins, which subsequently showed proliferation of the retinal pigment $\vec{N}$ associated with a scotoma, developed after contusion injuries. This appearance was $\frac{\circ}{7}$ said to be due to ischaemia brought on by rupture of the posterior ciliary arteries; somewhat similar appearances were produced experimentally in animals by section of the posterior ciliary arteries.

The cases reported above demonstrate a peculiar ophthalmoscopic appearance after a contusion injury to the eye. Cases $\mathrm{I}$ to 7 showed a limited choroidal lesion either around the disc (Cases I, 2, 3, 6, and 7) or extending from the disc region (Cases 4 and 5). An extension of the lesion to involve the macular area is seen in Cases I, 6, and 7. The choroidal lesion is usually an atrophic patch irregularly bespattered with pigment, and varying in colour from black to grey or greenish-grey. In all cases, the retinal vessels passed uninterruptedly over the choroidal atrophic patch.

Most of the patients were seen a long time (from I to Io years) after the injury, and therefore show the final and permanent fundus picture. Only in Case $\mathrm{I}$ were both the very early stage of the choroidal lesion and the late result observed. In all cases there was no other similar lesion in the same or in the contralateral one, and in every case there was a definite history of trauma followed by blurring of vision. No further investigations have been carried out because the defective vision and ophthalmoscopic appearances were related to the trauma. Treatment was ineffective and a permanent visual defect was the ultimate result.

Only in Case 8 was the condition mistaken for a central chorio-retinitis because the retina was involved, but the true cause was undetermined. This case is the only example of a retinal lesion due to injury to a vascular choroidal structure. The shape and the site of the lesion point to the involvement of an isolated cilio-retinal artery.

Because all these cases are directly related to a concussion injury and most of them show a peripapillary pigmented atrophic choroidal lesion and some a similar triangular or rectangular area in close contact with the disc margin, it appears that the lesion is due to a vascular derangement either of the circle of Zinn or of a short posterior ciliary artery. In Case 8 there was possibly an isolated cilio-retinal artery injury.

Treacher Collins (I9I7) believed that concussion injuries produced subchoroidal haemorrhage. The rupture of a short posterior artery is a plausible hypothesis for the production of such a haemorrhage.

Ashton (1952) suggested that capillary damage might be brought about primarily by 
an increase in the normal tissue pressure within a localized region of the choroid. A likely cause for such an increase in pressure is a haemorrhagic or inflammatory extravasation within the choroid; this exerts a marked effect on the choroidal vascular bed owing to the limitations imposed by the normal force of the intraocular pressure acting against the unyielding sclera.

The sequence of events following a concussion injury to the eye may be visualized as follows. The injury causes the posterior portion of the sclera to expand. This expansion may cause the rupture or occlusion of the vessels which perforate it perpendicularly or obliquely. A vascular channel like the circle of Zinn which is embedded in the sclera is more susceptible to such a lesion. Haemorrhage due to the rupture may cause secondary occlusion of the affected or neighbouring vessels. The haemorrhage or infarction which follows the secondary occlusion causes the characteristic darkening of the area supplied by the affected vessel, as in Case I. When the condition subsides, atrophic degenerative changes set in, giving the characteristic appearance seen in all the cases. There is a regular well-defined area of choroidal atrophy with pigment bespattered irregularly either over the whole region or at its periphery. The fact that the retinal vessels pass over the affected region without interruption means that the retina is not involved in the primary lesion, but the scotomata which develop in most cases indicate that at least the posterior neuro-epithelial layers of the retina are secondarily affected.

Because the macula is involved in most cases, it is apparent that this region is supplied by a branch arising either from the circle of Zinn or from a short ciliary artery.

A similar peripapillary choroidal atrophy with pigmentary disturbance is often present in high myopia (Fig. 10), and this suggests the occurrence of some similar vascular derangement, probably a disturbance of the blood supply through the stretching of the posterior sclera, a constant process in the progressive type of this condition. There may thus be a vascular basis for the atrophic changes present in high myopia.

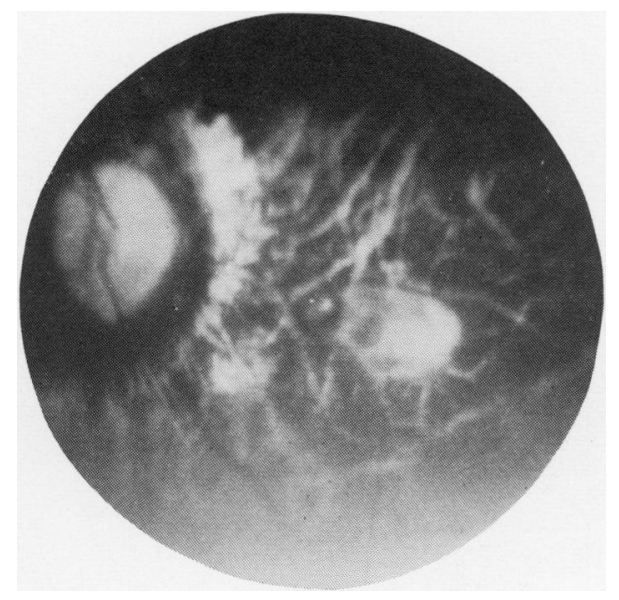

FIG. Io Peripapillary choroidal atrophy with pigment deposition in a case of high myopia.

\section{Summary}

Eight cases are reported of peripapillary choroidal atrophy following trauma. Two other cases with the same ophthalmoscopic appearances were found in the literature. Injury to the circle of Zinn or to a short posterior ciliary artery may be due to expansion of the posterior sclera after concussion trauma to the eye. The relationship of this hypothesis to the segmental arrangement of the blood supply to the choroid is discussed. 


\section{References}

Ashton, N. (1952) Brit. 7. Ophthal., 36, 465

Bartolozzi, T. (1952) Amer. F. Ophthal., 35, i 700

BONAMOUR, G., BRÉGEAT, P., BONNET, M., and JUGE, P. (1968) “La papille optique", p. 5 Io. Masson, Paris

COATs, G. (1907) Trans. ophthal. Soc. U.K., 27, 135

COLLINS, E. TREACHER (1916) Ibid., 36, 204

DUKE-ELDER, s. (1954) “Text-book of Ophthalmology", vol. 6, p. 5839. Kimpton, London.

and WYBAR, K. (I96I) "System of Ophthalmology", vol. 2. Kimpton, London.

KNAPP, H. (1868) v. Graefes Arch. Ophthal., 14, pt I, p. 245

MARIN-AMAT (1952) Discussion of Bartolozzi (see above)

RENARD, G., LEMASSON, C., and SARAuX, H. (1965) "Anatomie de l'oeil et de ses annexes", p. ioo. Masson, Paris

WYBAR, K. c. (1954) Brit. F. Ophthal., 38, $5^{1} 3$ 